\title{
Interpreting heterogeneity in intestinal tuft cell structure and function
}

\author{
Amrita Banerjee, ${ }^{1,2}$ Eliot T. McKinley, ${ }^{1,3}$ Jakob von Moltke, ${ }^{4}$ Robert J. Coffey, ${ }^{1,3}$ and Ken S. Lau ${ }^{1,2}$ \\ ${ }^{1}$ Epithelial Biology Center, Vanderbilt University Medical Center, Nashville, Tennessee, USA. ²Department of Cell and Developmental Biology, Vanderbilt University School of Medicine, Nashville, Tennessee, \\ USA. ${ }^{3}$ Department of Medicine, Vanderbilt University Medical Center, Nashville, Tennessee, USA. ${ }^{4}$ Department of Immunology, University of Washington, Seattle, Washington, USA.
}

\begin{abstract}
Intestinal tuft cells are a morphologically unique cell type, best characterized by striking microvilli that form an apical tuft. These cells represent approximately $0.5 \%$ of gut epithelial cells depending on location. While they are known to express chemosensory receptors, their function has remained unclear. Recently, numerous groups have revealed startling insights into intestinal tuft cell biology. Here, we review the latest developments in understanding this peculiar cell type's structure and function. Recent advances in volumetric microscopy have begun to elucidate tuft cell ultrastructure with respect to its cellular neighbors. Moreover, single-cell approaches have revealed greater diversity in the tuft cell population than previously appreciated and uncovered novel markers to characterize this heterogeneity. Finally, advanced model systems have revealed tuft cells' roles in mucosal healing and orchestrating type $\mathbf{2}$ immunity against eukaryotic infection. While much remains unknown about intestinal tuft cells, these critical advances have illuminated the physiological importance of these previously understudied cells and provided experimentally tractable tools to interrogate this rare cell population. Tuft cells act as luminal sensors, linking the luminal microbiome to the host immune system, which may make them a potent clinical target for modulating host response to a variety of acute or chronic immune-driven conditions.
\end{abstract}

\section{Introduction}

For close to a century, tuft cells (also known as brush or caveolated cells) have been identified in numerous epithelial tissues, including the gallbladder (1-5), stomach (4, 6-8), lung alveolus (9-13), and intestine (14-19). Decades of investigation have revealed little regarding the function of this mysterious cell type, until recently (20-24). This review focuses on recent breakthroughs into the biology and function of intestinal tuft cells (25-28). Tuft cells represent approximately $0.5 \%$ of epithelial cells in the murine small intestine and colon but are slightly more prevalent in the distal part of the small intestine, compared with the proximal (29). Tuft cells originate from $\mathrm{Lgr}^{+}$stem cells, similar to other differentiated intestinal epithelial cells (26), and are marked by doublecortin-like kinase 1 (DCLK1) (30). As DCLK1 ${ }^{+}$tuft cells were often observed in the quiescent " +4 " position of the intestinal crypt, they were originally considered a reserve stem cell pool (31-33). However, Gerbe et al. demonstrated that DCLK1 ${ }^{+}$tuft cells are a separate and distinct fifth intestinal epithelial cell lineage (30).

This review explores new insights into intestinal tuft cell structure enabled by advances in electron microscopy techniques. Furthermore, more sensitive, single-cell sequencing approaches have provided new understanding of the intestinal tuft cell gene signature, which may expand our current knowledge regarding tuft cell heterogeneity and function. Finally, we discuss recent reports on the role of intestinal tuft cells in (a) recovery of the intestinal epithelium from damage, and (b) induction of a type 2 immune response to eukaryotic intestinal colonization.

Conflict of interest: The authors have declared that no conflict of interest exists. Reference information: J Clin Invest. 2018;128(5):1711-1719.

https://doi.org/10.1172/JCl120330.

\section{Tuft cell morphology}

Early studies describing tuft cells in rodent models noted the distinctive apical bristles that formed a highly organized brush border, giving these cells their eponymous tufted morphology (1, $2,11,28,34)$. The distribution and dimensions of tuft cell microvilli, summarized in Table 1 , are profoundly different from those of neighboring enterocytes $(35,36)$. Unlike enterocytes, tuft cells do not possess a terminal web at the base of apical microvilli and possess a thinner fucose-rich glycocalyx above their apical membrane $(15,21,28)$. The shape of the main tuft cell body may vary depending on the organ $(1,21)$. Intestinal tuft cells have a cylindrical cell body that narrows at the apical and basal ends $(37,38)$, whereas alveolar tuft cells are flatter (9-13), and gallbladder tuft cells are cuboidal in shape $(4,5)$. These differences may reflect different environments or indicate organ-specific functions, but may also be experimental artifacts due to varying sectioning or fixation techniques.

Lateral projections from the tuft cell's basolateral membrane associate with neighboring cell nuclei $(1,2)$. As early as 1979, Luciano and Reale presented evidence of microvilli at the lateral cell border that appeared to continue into the cytoplasm of adjacent cells (1). However, limitations of conventional transmission electron microscopy could not provide the resolution necessary to characterize these protrusions, recently termed "cytospinules" by Hoover et al. (Figure 1 and ref. 36). Each tuft cell possesses 3-4 such projections, and a cytospinule can pierce the lateral membrane of a neighboring cell, making direct contact with its nuclear membrane (36). While the point of connection appears electron dense and its exact purpose remains unclear, it may be postulated that this tuft cell-to-neighboring cell contact serves as a direct means of communication or cargo transport. 


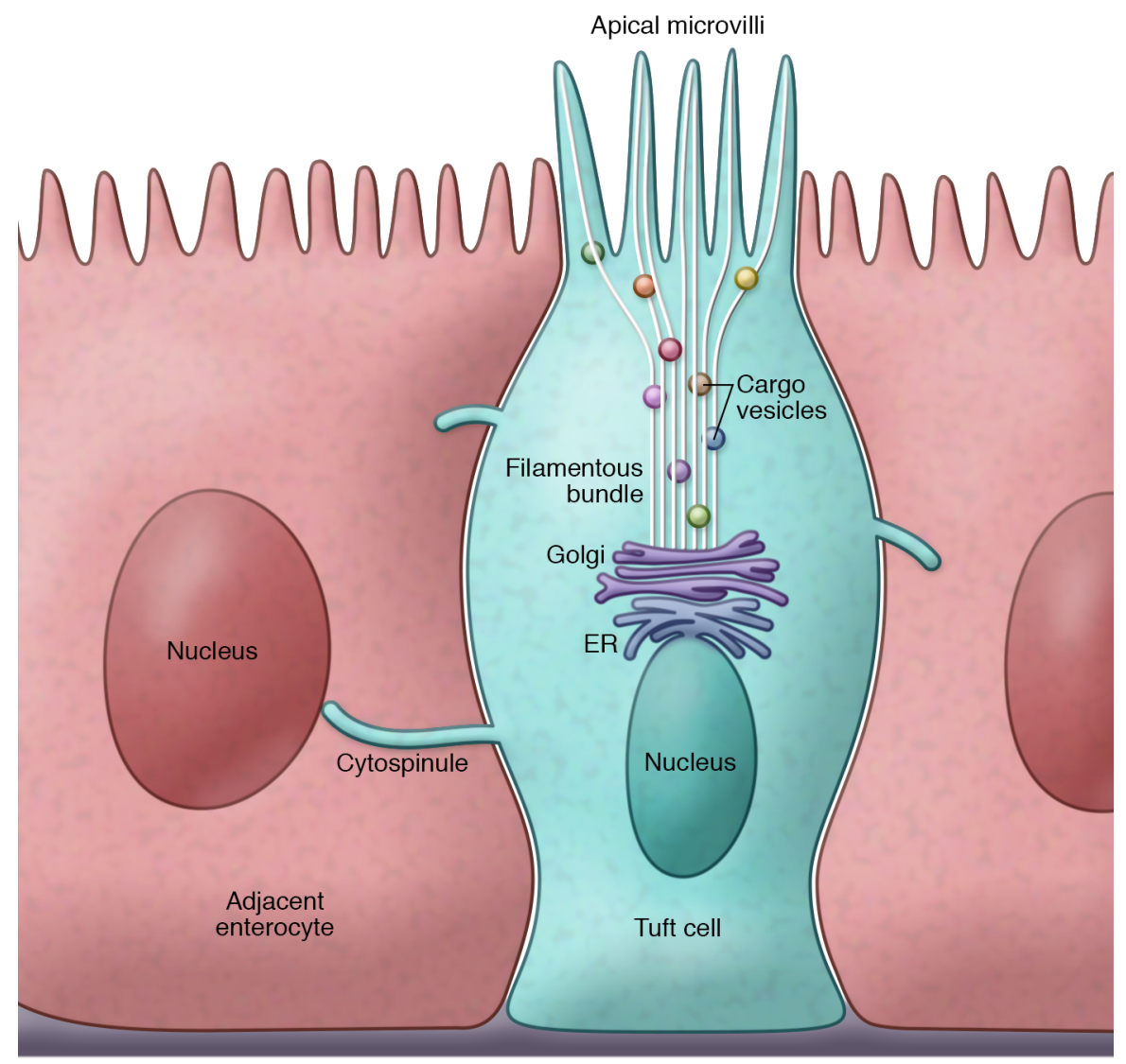

Basal lamina
Figure 1. Structural characteristics of intestinal tuft or caveolated cells. Intestinal tuft cells are easily distinguishable from neighboring enterocytes by their unique apical brush border and oval-shaped cell body. The apical microvilli connect the extracellular environment of the lumen to the intracellular cytoplasm via a filamentous bundle. Vesicles carrying unknown cargo are interspersed within the filamentous bundle, which terminates at a tubular network at the apex of the tuft cell nucleus. Lateral membrane projections or cytospinules emanate from the tuft cell and pierce the membrane of adjacent enterocytes. Cytospinules have been shown to directly contact the nuclei of tuft cel neighbors, possibly serving as a means of cellto-cell communication.

\section{Intracellular tuft cell structure}

Transmission electron microscopy studies in the epithelial lining of the rat submandibular gland were among the first to characterize the tuft cell intracellular landscape (39-42). These studies identified a filamentous bundle emanating from the apical microvilli and terminating at the ER $(21,39,40)$. The filamentous bundle was interspersed with transparent vesicles and electron-dense

\section{Table 1. Dimensions of enterocyte and tuft cell microvilli in mouse and human small intestine}

\begin{tabular}{|c|c|c|}
\hline Cell type & Murine small intestine ${ }^{\mathrm{A}}$ & Human small intestine ${ }^{\mathrm{B}}$ \\
\hline Enterocyte & $\begin{array}{c}0.132 \mu \mathrm{m}(\mathrm{SD} \pm 0.010 \mu \mathrm{m}) \\
\times 0.975 \mu \mathrm{m}(\mathrm{SD} \pm 0.101 \mu \mathrm{m})\end{array}$ & $\begin{array}{c}0.09 \mu \mathrm{m}(25 \text { th-75th percentile: } \\
0.09-0.10 \mu \mathrm{m}) \times 1.0 \mu \mathrm{m} \\
\text { (25th-75th percentile: } 0.8-1.1 \mu \mathrm{m})\end{array}$ \\
\hline Tuft cell & $\begin{array}{c}0.187 \mu \mathrm{m}(\mathrm{SD} \pm 0.024 \mu \mathrm{m}) \\
\times 2.289 \mu \mathrm{m}(\mathrm{SD} \pm 0.222 \mu \mathrm{m})\end{array}$ & $\begin{array}{c}0.12 \mu \mathrm{m}(25 \text { th- } 75 \text { th percentile: } \\
0.11-0.17 \mu \mathrm{m}) \times 1.7 \mu \mathrm{m}(25 \text { th-75th } \\
\text { percentile: } 1.4-2.0 \mu \mathrm{m})\end{array}$ \\
\hline
\end{tabular}

${ }^{A}$ Small intestinal tuft cells ( $n=3$ animals, 3 tuft cells/animal) analyzed using serial block-face scanning electron microscopy and automated tapecollecting ultramicrotome scanning electron microscopy. Width (thickness) and height (length) of microvilli from tuft cells and adjacent enterocytes were measured for comparison (36). ${ }^{\mathrm{B} S m a l l}$ intestinal tuft cells $(n=6)$ from more than 300 human biopsy specimens analyzed by transmission electron microscopy. Width (thickness) and height (length) of microvilli from tuft cells and adjacent enterocytes were measured using a micrometer (35). spheres containing indeterminate cargo $(21,39,40)$. More recent studies using volumetric electron microscopy offered increased resolution into the cytoplasmic contents of intestinal tuft cells (36, 43). Using a ChAT:GFP::Pyy-Cre:TdTomato transgenic mouse, Hoover et al. identified choline acetyltransferase ${ }^{+}\left(\mathrm{ChAT}^{+}\right)$tuft cells in the intestine (44) that were distinguishable from peptide YY-secreting (PYY-secreting) enteroendocrine cells (36, 43). Volumetric electron microscopy analysis of the filamentous bundle confirmed the presence of tubules running from the base of the apical microvilli to the ER (36). Electron-dense vesicular bodies of greater than $30 \mathrm{~nm}$ in diameter were interspersed within the filamentous bundle, possibly serving as a means of cargo transport between the ER and apical membrane (36). However, the cargo carried within those vesicles remains poorly characterized.

Tuft cells express chemosensory proteins, such as TRPM5 and $\alpha$-gustducin, which suggests that tuft cells are innervated by neighboring neurons. Structural studies have long attempted to characterize the relationship between these two cell types (36, $43,45-49)$. Studies in the rat submandibular gland noted that terminal nerve processes were often in close contact with tuft cells (20-25 nm in separation) $(39,40)$. Several studies in the mouse intestine also demonstrated close proximity between tuft cells and nerve cells (49). However, volumetric electron microscopy analysis by Hoover et al. did not identify any direct point of contact between tuft cells and nearby nerve cells (36). Transmission electron microscopy of human duodenojejunal tissue confirmed that human and mouse tuft cells share similar features, including the tufted micro- 
villar morphology, filamentous core, and lateral membrane projections (35). Interestingly, this human-based study observed direct contact between unmyelinated fibers of a mature neuron and the basolateral surface of a nearby tuft cell (35). While synaptic vesicles containing electron-dense granules were clearly apparent in the terminal axons, no evidence of a synapse was observed (35). However, in 300 human biopsy specimens, only six tuft cells were characterized and only a single tuft cell was associated with a naked terminal axon (35). As tuft cell rarity limits many structural studies, whether direct tuft cell-to-nerve cell contacts are a critical component of tuft cell biology remains an open question.

Tuft cells have also been shown to share common structural features with chromogranin A-expressing (CHGA-expressing), chemosensory enteroendocrine cells in the small and large intestine $(19,43$, 50-52). Enteroendocrine cells regulate nutrient sensory functions in the intestine and secrete a wide variety of neuropeptides, including PYY and cholecystokinin (CCK) but, like tuft cells, are epithelial in origin $(51,53,54)$. Like tuft cells, these cells are characterized by a tightly organized apical brush border but, unlike tuft cells, they possess a basal projection known as a neuropod (43). Neuropod projections are thought to associate with processes from enteric glia in the lamina propria (43), although direct contact is yet undocumented. Nonetheless, intestinal enterochromaffin cells were observed to form synaptic-like connections with nerve fibers (52). Therefore, based on structural studies, tuft and enteroendocrine cells may share some common physical characteristics that implicate these cell types in a sensory role within the intestinal epithelium $(26,52$ 54). As discussed below, tuft cells may also share a gene signature and progenitor cell with enteroendocrine cell subsets, further supporting the possibility of a relationship between the two cell types.

\section{Small intestinal tuft cell specification}

Investigations of intestinal epithelial specification initially classified tuft cells into the secretory lineage along with goblet, Paneth, and enteroendocrine cells (55-57). Atonal homolog 1 (Atoh1) is a basic helix-loop-helix transcription factor known as the master transcription factor regulator for secretory lineage specification $(56,58,59)$. In one study, Villin ${ }^{\text {CrEERT2-mediated intestine- }}$ specific knockout of Atoh1 eliminated $\mathrm{MUC2}^{+}$goblet, lysozyme ${ }^{+}$ Paneth, and DCLK1 ${ }^{+}$tuft cells (26). However, other groups utilizing similar Villin ${ }^{\text {Cre }}$ drivers or a stem cell-driven Lgr $5^{\text {EGFP-IRES-CreERT2 }}$ model demonstrated that tuft cells are preserved after Atoh1 loss $(53,60)$. In addition, a recent study further challenged the dependency of small intestinal tuft cell specification on Atoh1. Herring et al. used p-Creode, a novel computational trajectory-mapping algorithm, on single-cell data to determine that small intestinal tuft cells do not share a trajectory with the Atoh1-dependent secretory lineage (61). They further demonstrated that Atoh1 knockout using a Lrig1 ${ }^{\text {CreERT2 }}$ stem cell-specific driver resulted in significantly increased small intestinal tuft cell numbers, despite the absence of secretory goblet and Paneth cells (61). These findings suggest that tuft cell specification may be more complex than previously supposed and could be driven by environmental factors.

Interestingly, tuft cell specification depends on genes canonically involved in taste signaling (Table 2). Pou domain class 2 (Pou2f3) is a homeodomain transcription factor necessary for the specification of sweet, umami, and bitter taste cells (48, 62-64).
Pou2f3-null mice have a deficient taste response but also lack tuft cells in epithelial tissues, including the intestine (62-64). The taste signal transduction proteins TRPM5 (45, 48, 49, 65-67) and $\alpha$-gustducin $(48,66,68-70)$ are expressed in DCLK1 ${ }^{+}$cells, and Trpm5-null mice have decreased numbers of intestinal tuft cells (67). Numerous groups have demonstrated that DCLK1 ${ }^{+}$tuft cells express the Wnt target gene Sox9, although experiments in the Villin $^{\text {Cre }}$ Sox $9^{f / / f l}$ model make little mention of the effect of Sox 9 loss on tuft cell distribution $(26,55,71)$. Prostaglandin synthesis pathway members, cyclooxygenases COX-1 and COX-2, colocalize with tuft cell markers $(26,29,61)$. Acetylated tubulin and phosphorylated-EGFR (p-EGFR[Y1068]), are enriched at the apical tuft region $(29,47,61)$. Expression of the $\operatorname{Lgr} 5$ stem cell marker has been observed in tuft cells $(29,72)$. Recent studies on small intestinal tuft cells during acute helminth infection in the intestine confirmed expression of multiple type 2 immunity-related proteins, such as IL-25 (51, 67, 73, 74). Utilizing multiplex immunofluorescence, Herring et al. confirmed that p-STAT6, which is necessary for type 2 immunity $(67,75,76)$, and DCLK1 colocalize in the small intestine, whereas colonic tuft cells, which are not known to participate in type 2 immune responses, are p-STAT6 negative (61). McKinley et al. identified broad tuft cell heterogeneity between the small intestinal and colonic tuft cell population based on multiple marker expression (29). These results suggest that multiple tuft cell states can result in response to multiple, diverse environmental cues.

\section{Tuft cell gene signature}

Recent developments in single-cell RNA sequencing (scRNA-Seq) have revealed further insights into tuft cell heterogeneity. Haber et al. applied droplet-based scRNA-Seq to characterize the transcriptome of the mouse small intestinal epithelium. Clustering analysis of scRNA-Seq data identified two distinct populations of mature tuft cells, categorized as tuft- 1 and tuft-2 (74). Whereas both groups expressed Dclk1, the tuft-2 cluster was enriched for immune-related genes, including Ptprc, which encodes the pan-immune marker CD45 (74). This unexpected finding was confirmed through single-molecule FISH, where some DCLK1expressing tuft cells coexpressed Ptprc mRNA (74). The tuft-1 cluster was enriched for neuronal genes, including Ptgs1, which is plausible since tuft cells express COX-1 and COX-2 (26, 29, 61, 74, 77). Perhaps indicative of their newly discovered role in mounting a type 2 immune response against parasitic helminths, tuft-1 and tuft- 2 cells expressed the type 2 cytokine Il25 and the type 2related cytokine receptors Il17rb, Il4ra, and Il13ra (74). In contrast, only tuft-2 expressed significant levels of the type 2-promoting cytokine Tslp (74).

While characterizing the transcriptome of $\mathrm{Bmil}^{-\mathrm{GFP}^{+}}$stem cells, Yan et al. utilized scRNA-Seq to reveal intriguing evidence regarding similarities between tuft cells and Neurod1- and Prox1expressing enteroendocrine cells (51). In vivo studies demonstrated that Prox1-expressing cells were capable of lineage-tracing entire crypts during homeostasis and following irradiation-induced injury $(51,78)$. Prox1-GFP ${ }^{+}$cells are enriched for enteroendocrine secretory products, including $C c k$ and $P y y$, and tuft cell markers, Dclk1 and Trpm5 (51). Immunostaining of small intestinal tissue confirmed that $\mathrm{CHGA}^{+}$enteroendocrine cells and $\mathrm{DCLK}^{+}$tuft 
cells expressed Prox1, suggesting that some of these cells may act as a quiescent stem cell pool, activated following tissue damage $(51,54,60)$. However, while some DCLK1 ${ }^{+}$tuft cells expressed Prox1, a subset of tuft cells was Prox1 negative, indicating further heterogeneity in the tuft cell population, with some resembling enteroendocrine cells while others were less similar.

\section{Colonic tuft cells}

While the specification and function of small intestinal tuft cells have been extensively studied, colonic tuft cells have been less rigorously investigated, and it cannot be assumed that lessons learned in the small intestine are transferrable to the large intestine. Accordingly, Herring et al. computationally determined that the specification programs controlling colonic and small intestinal tuft cells may vary. As previously discussed, while Atoh1-deficient mice developed tuft cell hyperplasia in the small intestine, the large intestine was largely absent of DCLK1 ${ }^{+}$tuft cells when Atoh1 was ablated (61). This result suggests that Atoh1 may be necessary for tuft cell specification in the colon, while its role in small intestinal specification is less clear. Colonic tuft cells may also be capable of responding to perturbation of the luminal environment, as McKinley et al. demonstrated that colonic tuft cells increased in germfree mice upon introduction of microorganisms, although the effect was transient (29). These lines of evidence suggest much remains to be understood regarding possible variation in specification and function of tuft cells in the small intestine versus the colon.

\section{Tuft cells in intestinal epithelial damage response}

Recent work has begun to shed light on tuft cell function and we will focus primarily on the role of these cells in (a) recovery of the intestinal epithelium from damage, and (b) induction of a type 2 immune response against eukaryotic intestinal colonization.

A well-established model of intestinal epithelial damage is total body irradiation of more than $8 \mathrm{~Gy}$, which triggers doublestranded DNA breaks followed by destruction of crypt units and villus atrophy $(79,80)$. During the recovery process, the small intestinal epithelium undergoes a tightly controlled program of cell death and proliferation, which occurs in two distinct waves 6 and 24 hours after irradiation (77). The effects of high irradiation are nonlethal and reversible, with wild-type animals recovering normal intestinal morphology within 1 to 2 weeks through a stem cell-driven process $(77,79)$.

Although deleting Dclk1 using a Villin ${ }^{\mathrm{Cr} /+} \operatorname{Dclk}^{\mathrm{fl} / \mathrm{l} l}$ model did not produce a discernible phenotype at homeostasis, irradiated animals without epithelial Dclk1 rarely survived beyond five days, whereas irradiated control mice recovered as expected (77). In the intestine, Villin ${ }^{\mathrm{Cre} /+} \mathrm{Dclk} \mathrm{1}^{\mathrm{fl} / \mathrm{fl}}$ animals failed to recover normal crypt-villus architecture and barrier function after irradiation, demonstrative of a defective regenerative process, although initial crypt proliferation was unimpaired, as determined by BrdU incorporation (77). Irradiated Villin ${ }^{\mathrm{Cr} /+} \mathrm{Dclk}^{\mathrm{fl} / \mathrm{fl}}$ intestinal tissue showed time-dependent downregulation of the stem cell markers Lgr5 and Bmil compared with wild-type animals (77). Whereas the previous study investigated epithelial loss of Dclk1, Westphalen et al. interrogated the role of $\mathrm{DCLK} 1^{+}$tuft cells in epithelial regeneration, using a $D c l k 1^{\text {CreERT2/+ }}$ Rosa $26^{\text {DTA }}$ model to specifically ablate Dclk1-expressing cells (60). Similar to the previous study, total body irradiation induced significant morbidity in induced Dclk1 $1^{\text {CreERT2/+ }}$ Rosa26 $6^{\text {DTA }}$ animals within 7 days, with significant crypt aberration and increased number of apoptotic cells (60). However, DCLK1 expression is not limited to epithelial cells, as DCLK1 and a closely related splice variant, DCX-like, play a role in neuronal migration during brain development (81-83). Therefore, ablating DCLK1-expressing neurons or neural progenitor cells in the intestinal stroma may have a deleterious effect on intestinal integrity, independent of tuft cell function. Using quantitative imaging, McKinley et al. demonstrated that tuft cells are resistant to mucosal atrophy after acute fasting, while the number of other intestinal epithelial cell types can be reduced by up to one-third of their original abundance (29). The persistence of tuft cells in this setting could indicate a potential functional relevance for this cell type in tissue recovery. Thus, DCLK1 ${ }^{+}$tuft cells have been shown to contribute to epithelial regeneration following damage. It was initially hypothesized that DCLK1 ${ }^{+}$tuft cells may act as a damage-activated reserve stem cell pool.

To verify if tuft cells exhibit a stem cell gene signature, Chandrakesan et al. developed a Dclk1 $1^{\text {CreERT2 }}$ Rosa26-YFP mouse line, where YFP was a reliable marker for DCLK1+ tuft cells (84). Sequencing of sorted $\mathrm{YFP}^{+}$cells showed enrichment of the stem cell gene Bmi1 and pluripotency factors Sox 2 and Klf4, but not Lgr5, in comparison with non-YFP-expressing intestinal epithelial cells (84). These cells also expressed cell cycle inhibitors, including $C d k n 1 a / p 21$, but are de-enriched for the cell cycle driver $C d k 1$, suggesting that they may act as a quiescent stem cell population that is activated upon genotoxic insult (84). May et al. had previously demonstrated that individual, FACS-isolated DCLK1 ${ }^{+}$ cells can produce spheroids in vitro (32). These spheroids, when implanted into immunocompromised mice, developed nodular structures, which expressed intestinal epithelial secretory and absorptive fate markers (32). While this would imply DCLK1 ${ }^{+}$crypt cells are capable of acting as stem cells, studies in the mammary epithelium have demonstrated that in vitro culture and transplantation may reprogram non-stem cells into a multipotent cell state, potentially confounding the in vivo relevance of this experiment $(85,86)$. Moreover, Westphalen et al., using a Dclk1 $1^{\text {CreERT2 }}$ Rosa26-LacZ reporter mouse line, did not observe an increase in lineage-tracing events following irradiation- or dextran sodium sulfate-induced small intestinal injury, arguing against a reserve stem cell role for DCLK1 ${ }^{+}$cells (60). Furthermore, Nakanishi et al. showed that postmitotic DCLK1 ${ }^{+}$tuft cells do not lineage-trace under homeostatic conditions, and only cancer cells that gain Dclk1 expression can act as cancer stem cells (87). Thus, while DCLK1 $1^{+}$tuft cells may modulate injury responses, they do not appear to be a damage-activated quiescent stem cell population.

Although DCLK1 ${ }^{+}$intestinal tuft cells may not act as stem cells upon epithelial injury, they may play a crucial role in mucosal recovery. Irradiated Villin ${ }^{\mathrm{Cr} /+}{ }^{+} \mathrm{Dclk} 1^{f / f l}$ animals exhibited dramatic reduction in the expression of pluripotency factors, including Oct4 and Klf4, and self-renewal pathways, including Notch and Akt/ $\operatorname{mTOR}(77,88)$. However, bulk lysate analysis precluded a consensus on whether these factors are downregulated in DCLK1 ${ }^{+}$cells or in actual stem cells through a non-cell autonomous mechanism $(77,88)$. In support of the latter, Villin ${ }^{\mathrm{Cr} /+} \mathrm{D}$ clk1 $1^{f / f l}$ animals showed lower levels of epithelial COX-2 and serum prostaglandin E2 
(PGE2) 24 hours after irradiation (88). DCLK1 ${ }^{+}$tuft cells highly express COX-1 and COX-2, and these enzymes process arachidonic acid into secreted PGE2. Dclk1 overexpression in colonic epithelial YAMC cells increased levels of intracellular COX-2 and secreted PGE2 (88). Myoshi et al. recently showed that PGE2 induces stem and progenitor cell proliferation, promoting tissue repair following epithelial damage (89). Without Dclk1, epithelial cells were de-enriched for cell cycle regulators cyclin E1 and cyclin D1 after damage (88). While the evidence remains circumstantial, tuft cells may be a major source of PGE2 and, thereby, involved in inducing stem cell proliferation and promoting postirradiation tissue recovery in a non-cell autonomous manner $(60,79,90)$.

In addition to stem cell-driven epithelial recovery, Dclk1 may also play a critical role in the damage response phase. Villin ${ }^{\mathrm{Cre} /+}$ $D c l k 1^{f / f l}$ crypts had a significantly higher number of TUNEL ${ }^{+}$cells and were positive for the apoptotic markers caspase-3 and -9 (88). While there are multiple mechanisms of postirradiation cell survival, DNA double-stranded break repair is predominantly mediated by the ATM serine/threonine kinase (79). In support of this mechanism, markers of the ATM-dependent repair pathway, including phosphorylated-ATM, $\gamma \mathrm{H} 2 \mathrm{AX}$, and BRCA1, decreased in the small intestine of irradiated Villin ${ }^{\mathrm{Cre} /+} \mathrm{Dclk} \mathrm{1}^{\mathrm{fl} / \mathrm{fl}}$ mice (88). Coimmunoprecipitation using an anti-DCLK1 antibody as bait demonstrated a direct protein-protein interaction between DCLK1 and ATM (88). Dclk1-overexpressing YAMC cells exposed to irradiation showed increased phosphorylation of ATM and H2AX, suggesting an enhanced DNA damage response, as well as increased colony formation, a metric of cell survival $(79,88)$. Thus, Dclk1-expressing cells may be important in both initial DNA damage-associated crypt loss and subsequent stem cell-driven recovery. However, it remains to be seen whether and how $\mathrm{DCLK} 1^{+}$tuft cells categorically orchestrate each of these processes. While Westphalen et al. used a diphtheria toxin-based strategy to induce DCLK1 ${ }^{+}$ tuft cell loss, Chandrakesan et al. relied on a Villin ${ }^{\text {Cre }}$ system to ablate Dclk1 in all intestinal epithelial cells, including stem and progenitor cells. Furthermore, impaired barrier function in the Villin $^{\text {Cre }}$ Dclk $^{f l / f l}$ model may exacerbate the effects of irradiationinduced damage, independent of $\mathrm{DCLK}^{+}$tuft cells, thus complicating the interpretation of these results $(77,88)$. The use of more targeted cell ablation strategies may provide more definitive evidence of tuft cell-specific functions.

\section{Tuft cells in gastrointestinal helminth infection}

In 2016, three separate publications demonstrated that tuft cells play a critical role in mounting a type 2 immune response against parasitic worm colonization of the intestine. Parasitic worms, or helminths, are multicellular metazoans and present a significant global infectious burden $(64,67,73,75)$. Helminth eggs or infectious larvae pass into human hosts via contaminated soil or water sources and often colonize the proximal small intestine, namely the duodenum and jejunum $(73,75)$. The rodent metazoan parasite Nippostrongylus brasiliensis is a robust model for studying helminth infection. $N$. brasiliensis are delivered via subcutaneous injection in larval form and migrate through the circulatory system to the lung, where they molt $(64,75)$. Eventually, they migrate to the trachea and are coughed up, only to be swallowed by the mouse host and delivered to the intestine. In the gastrointestinal tract, the parasite reaches sexual maturity and produces eggs that develop into the adult form within a short period of time (75). Adult worms are expelled from the intestine 1 to 2 weeks following the initial infection. Host detection of parasitic worms induces a rapid type 2 immune response and subsequent remodeling of the intestinal epithelium via goblet cell hyperplasia $(64,67,73)$. Goblet cells produce the anti-helminth molecule resistin-like $1 \beta$ (RETN1 $\beta)$ in a type 2-dependent manner which, together with increased mucus production and smooth muscle hypercontractility, contributes to worm expulsion $(23,64,75)$.

Each of the three groups independently detected expansion of the intestinal tuft cell population following helminth colonization. Gerbe et al. and von Moltke et al. utilized N. brasiliensis and Heligmosomoides polygyrus, which are cleared by a type 2 immune response. Howitt et al. observed the same phenotype with these two worm types as well as Trichinella spiralis and the mouse protist Tritrichomonas muris. In all three studies, hyperplastic tuft cells in infected mice expressed canonical tuft cell markers, including DCLK1, SOX9, and COX-1, confirming that these were bona fide tuft cells and not an alternative, damageinduced cell type $(64,67,73)$. The investigators tested the necessity of intestinal tuft cells in driving worm expulsion using animal models deficient in tuft cell number or function $(64,67$, 73). As previously mentioned, Pou2f3-null mice lack intestinal tuft cells and tuft cell hyperplasia was absent in Pou2f3-null mice following $N$. brasiliensis infection (22). While wild-type animals recovered by day 9 or 10 after infection, Pou $2 f 3$-null mice had significant worm burden at postinfection day 42 and worms were detected in distal regions of the intestine, beyond their normal areas of adherence (64). Neither gustducin- nor Trpm5-null mice exhibited significant tuft cell expansion in the presence of $T$. muris compared with infected wild-type animals (67). Therefore, functional chemosensation within intestinal tuft cells appears necessary for detecting eukaryotic infection and mounting an effective immune response.

The type 2 cytokine IL- 25 plays a critical role in the tuft cell response to eukaryotic infection. Gerbe et al. identified an increase in mucosal IL-25 by RNA-Seq and its tuft cell-specific expression by immunohistochemistry at day 9 after infection. von Moltke et al. utilized a knockin IL-25-RFP-expressing mouse and showed constitutive IL-25 expression in intestinal tuft cells but not in other intestinal epithelial cell types. Similarly, Howitt et al. performed RT-qPCR on sorted tuft cells and showed Il25 enrichment compared with other epithelial subpopulations. All three groups confirmed that tuft cells are not a critical source of either TSLP or IL-33, two other type 2 cytokines known to be important in helminth response, although bulk analysis of tuft cells may have masked the subset-specific expression of Tslp revealed by single-cell analysis (51). A Villin ${ }^{\mathrm{Cr} /+} \mathrm{Il} 25^{\text {F25/F25 }}$ model enabled IL-25 ablation from epithelial cells and these mice lacked tuft cell hyperplasia on day 7 after $N$. brasiliensis infection (73). Furthermore, IL-25 did not act directly on epithelial cells to promote tuft cell expansion, based on in vitro intestinal organoid experiments (64). While von Moltke et al. showed similar results in in vitro studies, exogenously applied IL-25 increased tuft cell numbers in vivo, confirming that IL-25 must indirectly stimulate tuft cell hyperplasia, likely via immune cell subpopulations. 
A

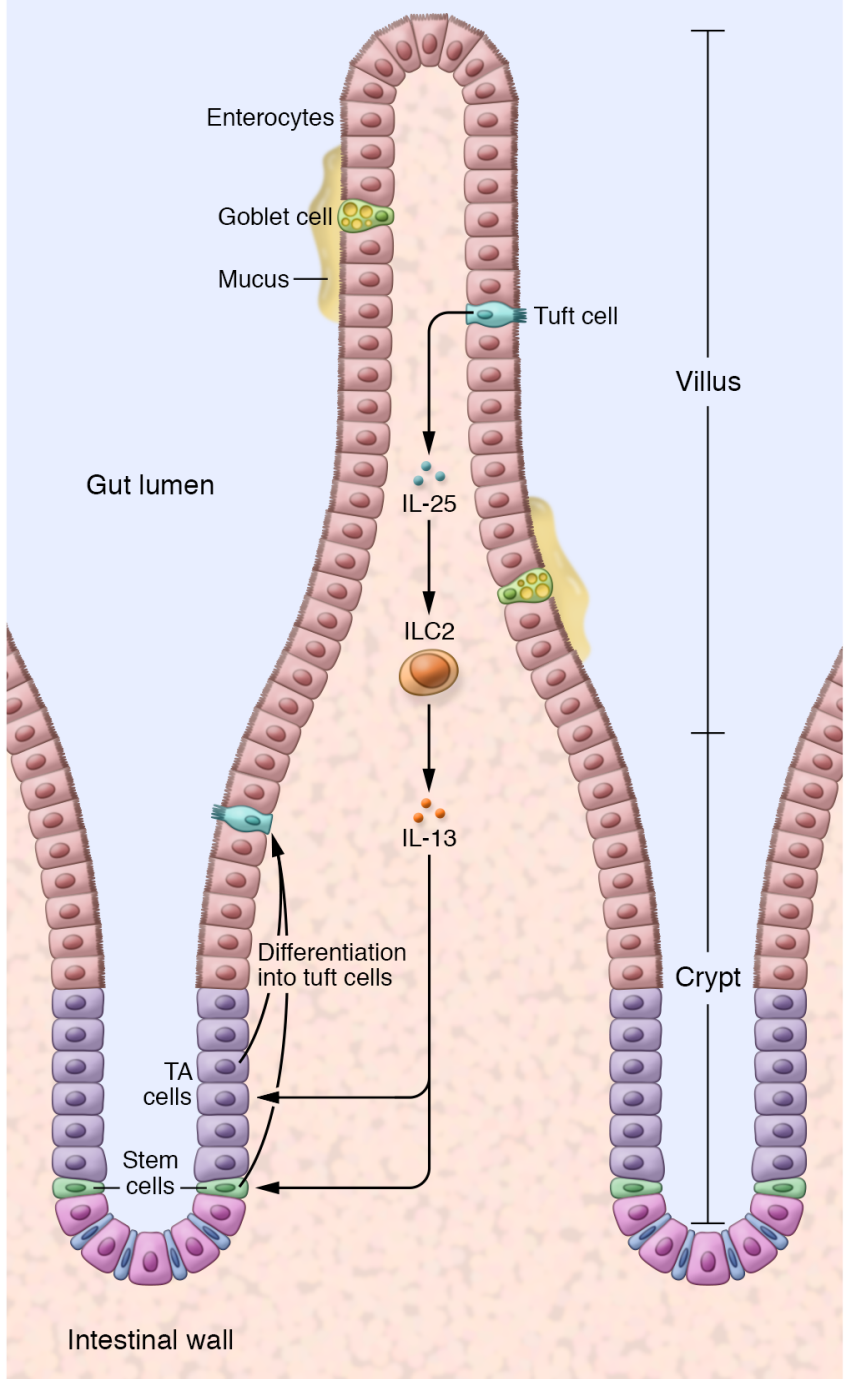

B

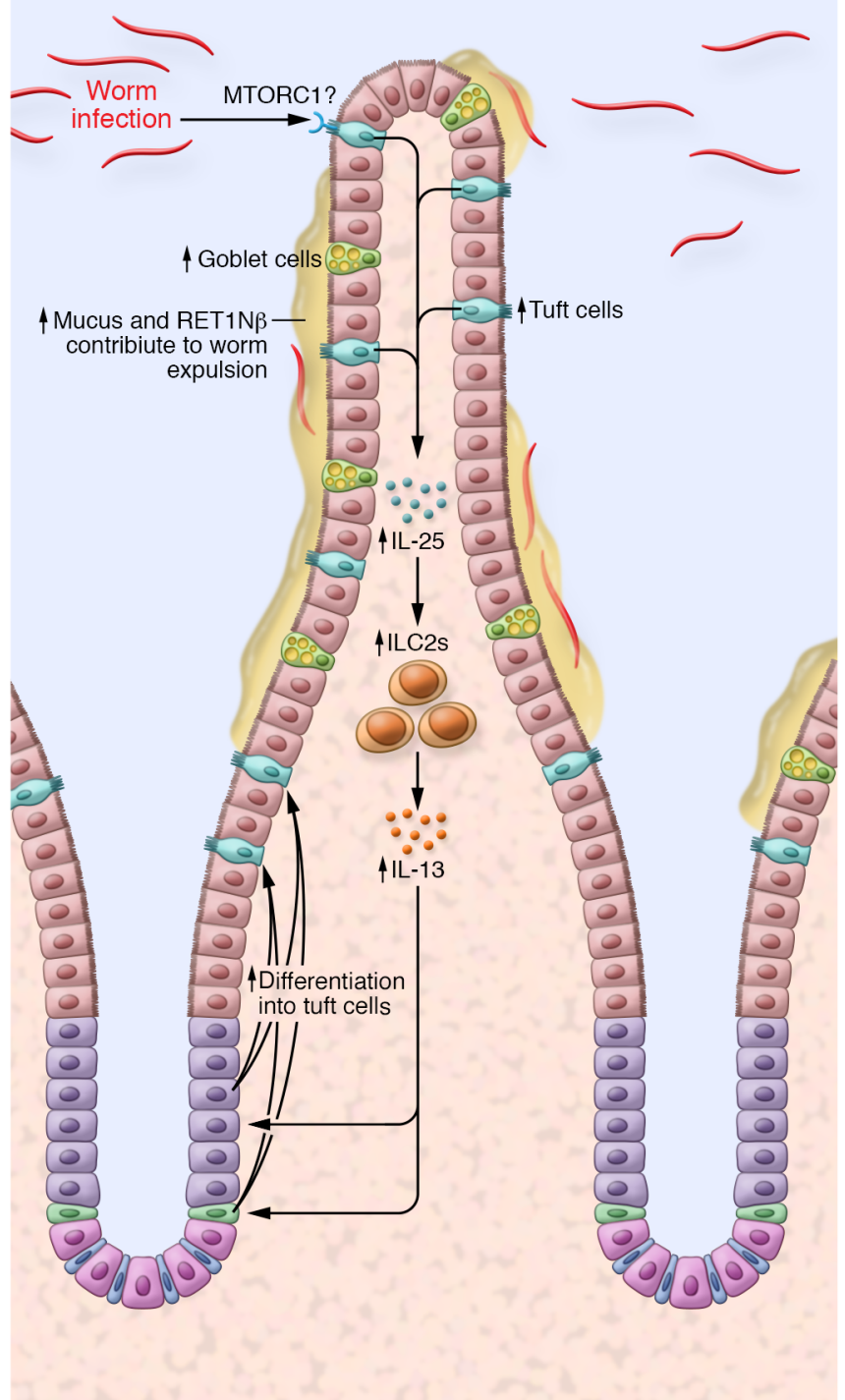

Figure 2. Small intestinal tuft cells induce a type $\mathbf{2}$ immune response following eukaryotic colonization of the gut. At homeostasis, DCLK1+ cells promote their own specification through release of IL-25, which stimulates ILC2s to produce IL-13. By an unknown mechanism, IL-13 stimulates stem cells and/ or transit-amplifying progenitors to differentiate into tuft cells. During eukaryotic colonization of the small intestine, tuft cells sense pathogens through an ill-defined mechanism, possibly involving the mTORC1 complex and Raptor. Increased IL-25 release by tuft cells drives IL-13-dependent expansion of the tuft cell lineage. Goblet cell hyperplasia and increased release of mucus as well as the anti-helminth molecule RETN1 $\beta$ contribute to worm expulsion from the proximal gut.

Previous literature confirmed that the type 2 cytokines IL-4 and IL-13 are involved in parasitic worm expulsion (75). von Moltke et al. demonstrated that exogenous administration of IL-4 in vivo and in ex vivo enteroid culture induces tuft cell expansion. However, $I l 4^{K N 2 / K N 2}$ animals, which are IL-4 deficient, still have a hyperplastic tuft cell phenotype following worm infection (73). Furthermore, Gerbe et al. observed that IL-4 levels were not significantly different between $N$. brasiliensisinfected wild-type and Pou2f3-null mice, despite the absence of tuft cells in the latter condition. These results implied that IL-4 is sufficient but not necessary for tuft cell expansion and its downstream immune response. In contrast, when Gerbe et al. and von Moltke et al. examined the effects of worm infection in an Il4ra-null model, which displays disrupted IL-4 and IL-13 signaling, they observed no tuft cell expansion (64, 73). Similarly, Il13 ${ }^{\mathrm{Cre} / \text { /re }} \mathrm{Gt}$ (Rosa26) $)^{\text {STOP-Flox:DTA/+ }}$ animals, which have disrupted IL-13 signaling, do not have tuft cell hyperplasia upon infection (73). In contrast to IL-4 levels, IL-13 levels decreased in N. brasiliensis-infected Pou2f3-null animals, compared with infected wild-type mice (64). Furthermore, in vivo and ex vivo addition of IL-13 was sufficient to induce tuft cell expansion $(64,67,73)$, implying IL-13 is necessary and sufficient for inducing tuft cell expansion by acting directly on the epithelium. Lineage-tracing experiments by von Moltke et al. indicated that, similar to homeostatic conditions, infectioninduced hyperplastic tuft cells are still specified via the $\mathrm{Lgr}^{+}$ stem cell population rather than by clonal expansion of the existing tuft cells or by transdifferentiation (73). These results 


\section{Table 2. Intestinal tuft cell markers and their proposed role in tuft cell function}

\begin{tabular}{|c|c|c|}
\hline Marker $^{A}$ & Proposed function ${ }^{B}$ & Reference \\
\hline Acetylated tubulin & Structural & 47 \\
\hline CK18 & Structural & 47,53 \\
\hline ChAT & Chemosensory & 43,44 \\
\hline$\alpha$-gustducin & Chemosensory & 48,68 \\
\hline TRPM5 & $\begin{array}{l}\text { Chemosensory, Type } 2 \\
\text { immune response }\end{array}$ & $48,49,53,67$ \\
\hline POU2F3 & $\begin{array}{l}\text { Chemosensory, Type } 2 \\
\text { immune response }\end{array}$ & 64 \\
\hline II- 25 & Type 2 immune response & $64,67,73,74$ \\
\hline p-STAT6 & Type 2 immune response & 61,67 \\
\hline COX-1, COX-2 & $\begin{array}{l}\text { Prostaglandin synthesis, } \\
\text { damage recovery }\end{array}$ & $26,29,49,53,61,77$ \\
\hline DCLK1 & $\begin{array}{l}\text { Quiescent stem cell, } \\
\text { damage recovery }\end{array}$ & $30-32,53$ \\
\hline HOPX & Unknown & 29,61 \\
\hline GFI1 $\beta$ & Unknown & 53 \\
\hline SOX9 & Unknown & $26,29,61$ \\
\hline p-EGFR(Y1068) & Unknown & 29,61 \\
\hline
\end{tabular}

${ }^{A}$ Based on antibody staining of intestinal tuft cells. ${ }^{B}$ Proposed role of protein marker within intestinal tuft cells, based on recent published literature.

suggest that IL-13 acts on stem or progenitor cell populations to drive tuft cell lineage expansion.

Th2 lymphocytes and innate lymphoid cells type 2 (ILC2s) both secrete IL-13, so that either or both cell populations could be the primary mediator of tuft cell expansion in response to eukaryotic infection (75). Th2 and ILC2 cell specification is controlled by transcription factor Gata3 but, unlike Th2 cells, ILC2s lack antigen receptors $(23,64,75)$. Elimination of both cell populations in immunodeficient RAG2;IL2R $\gamma$-null mice attenuated tuft cell hyperplasia following T. muris infection (67). T. muris-infected Rag2-null and N. brasiliensis-infected Rag-null mice, which lack Th2s but not ILC2s, had significant tuft cell expansion, confirming that Th2 cells are largely dispensable for this phenotype $(64,67)$. Tuft cell hyperplasia was not observed in infected Il17ra-null and Il2rg-null mice, which lack all innate lym-

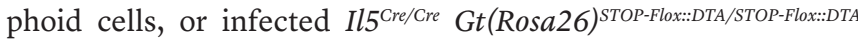
mice, which lack IL-5 $5^{+}$ILC2s, confirming that ILC2s are necessary to facilitate IL-25-induced tuft cell expansion, at least in these models of acute eukaryotic infection (73). Together, these studies established a feedback model, summarized in Figure 2, in which tuft cells, through the release of IL-25, induce ILC2 recruitment or expansion and IL-13 release, stimulating tuft and goblet cell hyperplasia.

While tuft cell taste receptors have been proposed to sense luminal infection, it is unclear how these signals are transduced in vivo, either through intracellular or intercellular means, to induce tuft cell hyperplasia. A recent report suggested a possible role for the mTOR complex 1 (mTORC1), a critical nutrient-sensing protein complex that drives cell proliferation and growth $(91,92)$. The mTORC1 complex includes the proteins mTOR, mLST8/GßL, PRAS40, DEPTOR, and Raptor, a scaffold protein necessary for
mTORC1 activation. Epithelial cell-specific Raptor ablation using Villin $^{\text {CreERT2/+ }}$ Raptor $^{\text {Af/l/l }}$ animals resulted in intestinal tuft cell loss, indicating that mTORC1 is necessary for homeostatic tuft cell lineage commitment (92). Previous studies have demonstrated that mTORC1 plays a role in specification of other intestinal epithelial cell types, as mTORC1 activation promotes goblet and Paneth cell specification $(93,94)$. Furthermore, tuft cell loss is accompanied by decreased epithelial IL25 mRNA and stromal IL13 mRNA expression, and lack of tuft cell expansion and type 2 immune response to T. muris infection (92). p-STAT6, which is required for type 2 immunity $(75,76)$, was decreased in infected Villin CreRT2/+ Raptor $^{\text {fl/ } / l}$ animals, likely due to inadequate IL-13 levels (92). Consequently, worm burden was significantly higher in infected

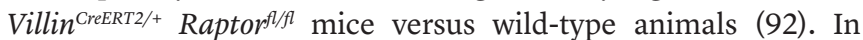
contrast to in vivo findings, ex vivo enteroids generated from Villin $^{\text {CreERT2 } /+}$ Raptor $^{\text {f/fl }}$ mice lacked a tuft cell defect or difference in IL-4 signaling (92). Thus, Raptor is unlikely to act in a cell autonomous fashion to direct tuft cell differentiation, but might be involved in intercellular communication. Thus, the direct mechanism linking luminal sensing to tuft cell response remains an important unanswered question for understanding how this chemosensory cell directs the clearance of eukaryotic parasites from the intestine.

\section{Concluding remarks}

While intestinal tuft cells have been poorly understood, a spate of recent articles has provided important insights into their structure and function. Technological strides in electron microscopy and scRNA-Seq have offered increased resolution of tuft cell ultrastructure and heterogeneity, respectively. However, though there is a greater appreciation for the extracellular structure of tuft cell cytospinules, it remains unclear what exact purpose these projections serve. Are they a means of direct cell-to-cell communication, and what, if any, are the functional consequences of cytospinule disruption? Similarly, single-cell analysis of small intestinal tuft cells has uncovered significant transcriptomic heterogeneity across this population, but does this diversity arise as a result of differential responses to luminal components or through intrinsic lineage-specification programs? Functionally, numerous publications have demonstrated that tuft cells do not appear to be stem cells under homeostatic or damage conditions, but is it possible that heterogeneity in gene and protein expression promotes functional specialization within this population?

Our current understanding of the tuft cell response to acute eukaryotic infection indicates that these cells may rely on chemosensory mechanisms to drive a type 2 immune response given the dampened tuft cell hyperplasia observed in infected Trpm5- and gustducin-null animals (67). However, the specific intracellular circuitry connecting tuft cell sensing of the luminal environment to intracellular response remains unclear. As tuft cells serve as a critical link between intestinal infection and downstream immunity, addressing these questions may have a significant clinical impact. Tuft cell function could be better leveraged to clear gastrointestinal pathogens or increase host resistance to infection, or even spur mucosal healing following epithelial damage. Stimulation of tuft cell secretory products could be used to redirect one 
variety of immune response to another in the context of inflammatory or allergic disease. Elucidation of tuft cell biology could provide new therapeutic strategies against a wide array of immunemediated conditions.

\section{Acknowledgments}

E.T. McKinley is funded by a training grant from the National Cancer Institute (NCI, grant R25CA092043). A. Banerjee and K.S. Lau are funded by the National Institute of Diabetes and Digestive and Kidney Diseases (R01DK103831 and P30DK058404) and the NCI (P50CA095103). J.V. Moltke is funded by the National Institute of Allergy and Infectious Diseases (DP2AI136596) and a Dale Frey Breakthrough Scientist Award from the Damon Runyon Cancer Research Foundation. R.J. Coffey is funded by the NCI (R35CA197570 and P50CA095103). The authors would like to thank the Vanderbilt Epithelial Biology Center for helpful discussions.

Address correspondence to: Ken S. Lau, Epithelial Biology Center, Vanderbilt University Medical Center, 2213 Garland Ave., 10475 MRB IV, Nashville, Tennessee 37232-0441, USA. Phone: 615.936.6859; Email:ken.s.lau@vanderbilt.edu.
1. Luciano L, Reale E. A new morphological aspect of the brush cells of the mouse gallbladder epithelium. Cell Tissue Res. 1979;201(1):37-44.

2. Luciano L, Reale E. Brush cells of the mouse gallbladder. A correlative light- and electron-microscopical study. Cell Tissue Res. 1990;262(2):339-349.

3. Luciano L, Reale E. Presence of brush cells in the mouse gallbladder. Microsc Res Tech. 1997;38(6):598-608.

4. Luciano L, Groos S, Reale E. Brush cells of rodent gallbladder and stomach epithelia express neurofilaments. J Histochem Cytochem. 2003;51(2):187-198.

5. Gilloteaux J, Pomerants B, Kelly TR. Human gallbladder mucosa ultrastructure: evidence of intraepithelial nerve structures. Am J Anat. 1989;184(4):321-333.

6. Kugler P, Höfer D, Mayer B, Drenckhahn D. Nitric oxide synthase and NADP-linked glucose6-phosphate dehydrogenase are co-localized in brush cells of rat stomach and pancreas. J Histochem Cytochem. 1994;42(10):1317-1321.

7. Luciano L, Armbruckner L, Sewing KF, Reale E. Isolated brush cells of the rat stomach retain their structural polarity. Cell Tissue Res. 1993;271(1):47-57.

8. Johnson FR, Young BA. Undifferentiated cells in gastric mucosa. J Anat. 1968;102(pt 3):541-551.

9. Meyrick B, Reid L. The alveolar brush cell in rat lung - a third pneumonocyte. JUltrastruct Res. 1968;23(1):71-80.

10. Foliguet B, Grignon G. [Type III pneumocyte. The alveolar brush-border cell in rat lung. Study by transmission electron microscopy (author's transl)]. Poumon Coeur. 1980;36(2):149-153.

11. Hijiya K, Okada Y, Tankawa H. Ultrastructural study of the alveolar brush cell. JElectron Microsc (Tokyo). 1977;26(4):321-329.

12. Hijiya K. Electron microscope study of the alveolar brush cell. J Electron Microsc (Tokyo). 1978;27(3):223-227.

13. DiMaio MF, Dische R, Gordon RE, Kattan M. Alveolar brush cells in an infant with desquamative interstitial pneumonitis. Pediatr Pulmonol. 1988;4(3):185-191.

14. Trier JS, Allan CH, Marcial MA, Madara JL. Structural features of the apical and tubulovesicular membranes of rodent small intestinal tuft cells. Anat Rec. 1987;219(1):69-77.

15. Isomaki AM. Electron microscope observations on a special cell type in the gastro-intestinal epithelium of some laboratory animals. Acta Pathol
Microbiol Scand A. 1962;154(suppl):115-118.

16. Isomäki AM. A new cell type (tuft cell) in the gastrointestinal mucosa of the rat. Acta Pathol Microbiol Scand A. 1973;240(suppl):1-35.

17. Carstens PH, Broghamer WL, Hire D. Malignant fibrillo-caveolated cell carcinoma of the human intestinal tract. Hum Pathol. 1976;7(5):505-517.

18. McNabb JD, Sandborn E. Filaments in the microvillous border of intestinal cells. JCell Biol. 1964;22:701-704.

19. Okamoto K, et al. Immunohistochemical and electron microscopic characterization of brush cells of the rat cecum. Med Mol Morphol. 2008;41(3):145-150.

20. Grencis RK, Worthington JJ. Tuft cells: a new flavor in innate epithelial immunity. Trends Parasitol. 2016;32(8):583-585.

21. Sato A. Tuft cells. Anat Sci Int. 2007;82(4):187-199.

22. Gerbe F, Legraverend C, Jay P. The intestinal epithelium tuft cells: specification and function. Cell Mol Life Sci. 2012;69(17):2907-2917.

23. Gerbe F, Jay P. Intestinal tuft cells: epithelial sentinels linking luminal cues to the immune system. Mucosal Immunol. 2016;9(6):1353-1359.

24. Middelhoff M, et al. Dclk1-expressing tuft cells: critical modulators of the intestinal niche? Am J Physiol Gastrointest Liver Physiol. 2017;313(4):G285-G299.

25. Höfer D, Drenckhahn D. Identification of brush cells in the alimentary and respiratory system by antibodies to villin and fimbrin. Histochemistry. 1992;98(4):237-242.

26. Gerbe F, et al. Distinct ATOH1 and Neurog3 requirements define tuft cells as a new secretory cell type in the intestinal epithelium. JCell Biol. 2011;192(5):767-780.

27. Gebert A, al-Samir K, Werner K, Fassbender S, Gebhard A. The apical membrane of intestinal brush cells possesses a specialised, but species-specific, composition of glycoconjugates - on-section and in vivo lectin labelling in rats, guinea-pigs and mice. Histochem Cell Biol. 2000;113(5):389-399.

28. Silva DG. The fine structure of multivesicular cells with large microvilli in the epithelium of the mouse colon. JUltrastruct Res. 1966;16(5):693-705.

29. McKinley ET, et al. Optimized multiplex immunofluorescence single-cell analysis reveals tuft cell heterogeneity. JCI Insight. 2017;2(11):93487.

30. Gerbe F, Brulin B, Makrini L, Legraverend C, Jay P. DCAMKL-1 expression identifies Tuft cells rather than stem cells in the adult mouse intestinal epithelium. Gastroenterology.
2009;137(6):2179-2180; author reply 2180.

31. May R, Riehl TE, Hunt C, Sureban SM, Anant S, Houchen CW. Identification of a novel putative gastrointestinal stem cell and adenoma stem cell marker, doublecortin and CaM kinase-like-1, following radiation injury and in adenomatous polyposis coli/multiple intestinal neoplasia mice. Stem Cells. 2008;26(3):630-637.

32. May R, et al. Doublecortin and CaM kinase-like-1 and leucine-rich-repeat-containing G-proteincoupled receptor mark quiescent and cycling intestinal stem cells, respectively. Stem Cells. 2009;27(10):2571-2579.

33. Dekaney CM, Gulati AS, Garrison AP, Helmrath MA, Henning SJ. Regeneration of intestinal stem/progenitor cells following doxorubicin treatment of mice. Am J Physiol Gastrointest Liver Physiol. 2009;297(3):G461-G470.

34. Nabeyama A, Leblond CP. "Caveolated cells" characterized by deep surface invaginations and abundant filaments in mouse gastro-intestinal epithelia. Am JAnat. 1974;140(2):147-165.

35. Morroni M, Cangiotti AM, Cinti S. Brush cells in the human duodenojejunal junction: an ultrastructural study. J Anat. 2007;211(1):125-131.

36. Hoover B, Baena V, Kaelberer MM, Getaneh F, Chinchilla S, Bohórquez DV. The intestinal tuft cell nanostructure in 3D. Sci Rep. 2017;7(1):1652.

37. Isomäki AM. Electron microscope observations on a special cell type in the gastro-intestinal epithelium of some laboratory animals. Acta Pathol Microbiol Scand A. 1962;154(suppl):115-118.

38. Isomäki AM. A new cell type (tuft cell) in the gastrointestinal mucosa of the rat. A transmission and scanning electron microscopic study. Acta Pathol Microbiol Scand A. 1973;suppl 240:1-35

39. Sato A, Hisanaga Y, Inoue Y, Nagato T, Toh H. Three-dimensional structure of apical vesicles of tuft cells in the main excretory duct of the rat submandibular gland. Eur J Morphol. 2002;40(4):235-239.

40. Sato A, Miyoshi S. Fine structure of tuft cells of the main excretory duct epithelium in the rat submandibular gland. Anat Rec. 1997;248(3):325-331.

41. Nevalainen TJ. Ultrastructural characteristics of tuft cells in mouse gallbladder epithelium. Acta Anat (Basel). 1977;98(2):210-220.

42. Sato A, Suganuma T, Ide S, Kawano J, Nagato T. Tuft cells in the main excretory duct of the rat submandibular gland. Eur J Morphol. 2000;38(4):227-231.

43. Bohórquez DV, Samsa LA, Roholt A, Medicetty S, Chandra R, Liddle RA. An enteroendocrine cell- 
enteric glia connection revealed by $3 \mathrm{D}$ electron microscopy. PLoS One. 2014;9(2):e89881.

44. Schütz B, et al. Chemical coding and chemosensory properties of cholinergic brush cells in the mouse gastrointestinal and biliary tract. Front Physiol. 2015;6:87.

45. Kaske S, et al. TRPM5, a taste-signaling transient receptor potential ion-channel, is a ubiquitous signaling component in chemosensory cells. BMC Neurosci. 2007;8:49.

46. Sbarbati A, Osculati F. The taste cell-related diffuse chemosensory system. Prog Neurobiol. 2005;75(4):295-307.

47. Höfer D, Drenckhahn D. Cytoskeletal markers allowing discrimination between brush cells and other epithelial cells of the gut including enteroendocrine cells. Histochem Cell Biol. 1996;105(5):405-412.

48. Bezençon C, le Coutre J, Damak S. Taste-signaling proteins are coexpressed in solitary intestinal epithelial cells. Chem Senses. 2007;32(1):41-49.

49. Bezençon C, et al. Murine intestinal cells expressing Trpm 5 are mostly brush cells and express markers of neuronal and inflammatory cells. JComp Neurol. 2008;509(5):514-525.

50. Tsubouchi S, Leblond CP. Migration and turnover of entero-endocrine and caveolated cells in the epithelium of the descending colon, as shown by radioautography after continuous infusion of ${ }^{3} \mathrm{H}$-thymidine into mice. Am J Anat. 1979;156(4):431-451.

51. Yan KS, et al. Intestinal enteroendocrine lineage cells possess homeostatic and injuryinducible stem cell activity. Cell Stem Cell. 2017;21(1):78-90.e6.

52. Bellono NW, et al. Enterochromaffin cells are gut chemosensors that couple to sensory neural pathways. Cell. 2017;170(1):185-198.e16.

53. Bjerknes $\mathrm{M}$, et al. Origin of the brush cell lineage in the mouse intestinal epithelium. Dev Biol. 2012;362(2):194-218.

54. Bjerknes M, Cheng H. Neurogenin 3 and the enteroendocrine cell lineage in the adult mouse small intestinal epithelium. Dev Biol. 2006;300(2):722-735.

55. Durand A, et al. Functional intestinal stem cells after Paneth cell ablation induced by the loss of transcription factor Math1 (Atoh1). Proc Natl Acad Sci U S A. 2012;109(23):8965-8970.

56. Shroyer NF, Helmrath MA, Wang VY, Antalffy B, Henning SJ, Zoghbi HY. Intestine-specific ablation of mouse atonal homolog 1 (Math1) reveals a role in cellular homeostasis. Gastroenterology. 2007;132(7):2478-2488.

57. Kazanjian A, Noah T, Brown D, Burkart J, Shroyer NF. Atonal homolog 1 is required for growth and differentiation effects of notch/gammasecretase inhibitors on normal and cancerous intestinal epithelial cells. Gastroenterology. 2010;139(3):918-28, 928.e1.

58. Han ME, Baek SJ, Kim SY, Kang CD, Oh SO. ATOH1 can regulate the tumorigenicity of gastric cancer cells by inducing the differentiation of cancer stem cells. PLoS One. 2015;10(5):e0126085.

59. VanDussen KL, et al. Notch signaling modulates proliferation and differentiation of intestinal crypt base columnar stem cells. Development. 2012;139(3):488-497.

60. Westphalen CB, et al. Long-lived intestinal tuft cells serve as colon cancer-initiating cells. JClin Invest. 2014;124(3):1283-1295.

61. Herring CA, et al. Unsupervised trajectory analysis of single-cell RNA-seq and imaging data reveals alternative tuft cell origins in the gut. Cell Syst. 2018;6(1):37-51.e9.

62. Matsumoto I, Ohmoto M, Narukawa M, Yoshihara Y, Abe K. Skn-1a (Pou2f3) specifies taste receptor cell lineage. Nat Neurosci. 2011;14(6):685-687.

63. Yamashita J, Ohmoto M, Yamaguchi T, Matsumoto I, Hirota J. Skn-1a/Pou2f3 functions as a master regulator to generate Trpm5-expressing chemosensory cells in mice. PLoS One. 2017;12(12):e0189340.

64. Gerbe F, et al. Intestinal epithelial tuft cells initiate type 2 mucosal immunity to helminth parasites. Nature. 2016;529(7585):226-230.

65. Oike $\mathrm{H}$, et al. Arachidonic acid can function as a signaling modulator by activating the TRPM5 cation channel in taste receptor cells. Biochim Biophys Acta. 2006;1761(9):1078-1084.

66. Gulbransen BD, Clapp TR, Finger TE, Kinnamon SC. Nasal solitary chemoreceptor cell responses to bitter and trigeminal stimulants in vitro. $\mathrm{J} \mathrm{Neu}$ rophysiol. 2008;99(6):2929-2937.

67. Howitt MR, et al. Tuft cells, taste-chemosensory cells, orchestrate parasite type 2 immunity in the gut. Science. 2016;351(6279):1329-1333.

68. Höfer D, Püschel B, Drenckhahn D. Taste receptor-like cells in the rat gut identified by expression of $\alpha$-gustducin. Proc Natl Acad Sci U S A. 1996;93(13):6631-6634.

69. Höfer D, Drenckhahn D. Identification of the taste cell G-protein, $\alpha$-gustducin, in brush cells of the rat pancreatic duct system. Histochem Cell Biol. 1998;110(3):303-309.

70. Jang $\mathrm{HJ}$, et al. Gut-expressed gustducin and taste receptors regulate secretion of glucagonlike peptide-1. Proc Natl Acad Sci U S A. 2007;104(38):15069-15074.

71. Mori-Akiyama Y, et al. SOX9 is required for the differentiation of paneth cells in the intestinal epithelium. Gastroenterology. 2007;133(2):539-546.

72. Itzkovitz S, et al. Single-molecule transcript counting of stem-cell markers in the mouse intestine. Nat Cell Biol. 2011;14(1):106-114.

73. von Moltke J, Ji M, Liang HE, Locksley RM. Tuft-cell-derived IL-25 regulates an intestinal ILC2-epithelial response circuit. Nature. 2016;529(7585):221-225.

74. Haber AL, et al. A single-cell survey of the small intestinal epithelium. Nature. 2017;551(7680):333-339.

75. Allen JE, Sutherland TE. Host protective roles of type 2 immunity: parasite killing and tissue repair, flip sides of the same coin. Semin Immunol. 2014;26(4):329-340.

76. Ramanan D, et al. Helminth infection promotes colonization resistance via type 2 immunity. Science. 2016;352(6285):608-612.

77. May R, et al. Brief report: Dclk1 deletion in tuft cells results in impaired epithelial repair after radiation injury. Stem Cells. 2014;32(3):822-827.

78. Yan KS, et al. The intestinal stem cell markers Bmi1 and Lgr5 identify two functionally distinct populations. Proc Natl Acad Sci U S A. 2012;109(2):466-471.

79. Booth C, Tudor G, Tudor J, Katz BP, MacVittie TJ. Acute gastrointestinal syndrome in high-dose irradiated mice. Health Phys. 2012;103(4):383-399.

80. Potten CS, Owen G, Booth D. Intestinal stem cells protect their genome by selective segregation of template DNA strands. J Cell Sci. 2002;115(pt 11):2381-2388.

81. Koizumi H, Tanaka T, Gleeson JG. Doublecortinlike kinase functions with doublecortin to mediate fiber tract decussation and neuronal migration. Neuron. 2006;49(1):55-66.

82. Koizumi H, Higginbotham H, Poon T, Tanaka T, Brinkman BC, Gleeson JG. Doublecortin maintains bipolar shape and nuclear translocation during migration in the adult forebrain. Nat Neurosci. 2006;9(6):779-786.

83. Verissimo CS, et al. Silencing of the microtubuleassociated proteins doublecortin-like and doublecortin-like kinase-long induces apoptosis in neuroblastoma cells. Endocr Relat Cancer. 2010;17(2):399-414.

84. Chandrakesan P, et al. Dclk $1^{+}$small intestinal epithelial tuft cells display the hallmarks of quiescence and self-renewal. Oncotarget. 2015;6(31):30876-30886.

85. Prater MD, et al. Mammary stem cells have myoepithelial cell properties. Nat Cell Biol. 2014;16(10):942-50, 1-7.

86. Van Keymeulen A, et al. Distinct stem cells contribute to mammary gland development and maintenance. Nature. 2011;479(7372):189-193.

87. Nakanishi Y, et al. Dclk1 distinguishes between tumor and normal stem cells in the intestine. Nat Genet. 2013;45(1):98-103.

88. Chandrakesan $P$, et al. Intestinal tuft cells regulate the ATM mediated DNA Damage response via Dclk1 dependent mechanism for crypt restitution following radiation injury. Sci Rep. 2016;6:37667.

89. Miyoshi H, et al. Prostaglandin E2 promotes intestinal repair through an adaptive cellular response of the epithelium. EMBO J. 2017;36(1):5-24.

90. Cohn SM, Schloemann S, Tessner T, Seibert $\mathrm{K}$, Stenson WF. Crypt stem cell survival in the mouse intestinal epithelium is regulated by prostaglandins synthesized through cyclooxygenase-1. J Clin Invest. 1997;99(6):1367-1379.

91. Laplante M, Sabatini DM. mTOR signaling in growth control and disease. Cell. 2012;149(2):274-293.

92. Aladegbami B, et al. Epithelial cell specific Raptor is required for initiation of type 2 mucosal immunity in small intestine. Sci Rep. 2017;7(1):5580.

93. Zhou Y, Rychahou P, Wang Q, Weiss HL, Evers BM. TSC2/mTORC1 signaling controls Paneth and goblet cell differentiation in the intestinal epithelium. Cell Death Dis. 2015;6:e1631.

94. van Es JH, et al. Notch/ $\gamma$-secretase inhibition turns proliferative cells in intestinal crypts and adenomas into goblet cells. Nature. 2005;435(7044):959-963. 